\title{
Satisfação da imagem corporal e suas relações com sexo biológico, orientação sexual, identidade de gênero e esquemas de gênero do autoconceito em bailarinos: uma revisão sistemática
}

\section{RESUMO}

O conceito de imagem corporal está baseado a partir de um construto multidimensional, formado das representações sobre o tamanho e aparência do corpo. Objetivou-se a verificar de forma sistematizada a literatura estudos que apresentassem a relação entre satisfação da imagem corporal e variáveis sexuais. Os resultados demonstraram que a área que estuda a percepção da imagem corporal em bailarinos ainda não percebeu a importância de outras dimensões como identidade de gênero, orientação sexual e os esquemas de gênero do autoconceito, que podem ter maior impacto do que apenas o critério sexo biológico. $\mathrm{O}$ sexo biológico ainda é a principal variável de controle na comparação nos estudos entre os bailarinos, sendo urgente a necessidade de outras dimensões da identidade a serem incorporadas nos próximos estudos, como as citadas acima, para ampliar a capacidade de explicarmos a satisfação corporal humana

PALAVRAS-CHAVE: Imagem corporal; Sexo; Orientação sexual; Identidade de gênero

\author{
Alex Carneiro Brandão \\ Mestre \\ Universidade Federal de Santa Catarina - UFSC \\ Florianópolis, Santa Catarina, Brasil \\ alexcarneiro.b@hotmail.com \\ (ㄱ) https://orcid.org/0000-0003-1358-719X
}

Kamyla Thais Dias de Freitas

Mestra

Universidade do Estado de Santa Catarina - UDESC

FAED/PPGE, Florianópolis, Santa Catarina, Brasil

kamyla.freitas@outlook.com

(1) https://orcid.org/0000-0002-0770-7952

Walan Robert da Silva

Mestre

Universidade do Estado de Santa Catarina - UDESC

CEFID/PPGCMH, Florianópolis, Santa Catarina,

Brasil

walanrobert@hotmail.com

https://orcid.org/0000-0003-0568-4272

\section{Helton Pereira De Carvalho}

Mestre

Universidade do Estado de Santa Catarina - UDESC

CEFID/PPGCMH, Florianópolis, Santa Catarina,

Brasil

helton-13@hotmail.com

https://orcid.org/0000-0001-9456-0436

Fernando Luiz Cardoso

Doutor

Universidade do Estado de Santa Catarina - UDESC

CEFID/PPGCMH e FAED/PPGE

Florianópolis, Santa Catarina, Brasil

fernandocardoso.ph.d.lagesc@gmail.com

(- https://orcid.org/0000-0002-3074-0988 
Satisfaction of body image and its relationships with biological sex, sexual orientation, gender identity and gender schemas of self-concept in dancers: a systematic review

\begin{abstract}
English abstract version the concept of body image is based on a multidimensional construct, formed of representations about the size and appearance of the body. The objective was to systematically verify the literature studies that showed the relationship between body image satisfaction and sexual variables. The results showed that the area that studies the perception of body image in dancers has not yet perceived the importance of other dimensions such as gender identity, sexual orientation and self-concept gender schemes, which may have greater impact than just the biological sex criterion. Biological sex is still the main control variable in the comparison among studies among dancers, and there is an urgent need for other dimensions of identity to be incorporated in future studies, such as those cited above, to increase the capacity to explain human body satisfaction.
\end{abstract}

KEYWORDS: Body image; Sex; Sexual orientation; Gender identity

Satisfacción de la imagen corporal y sus relaciones con sexo biológico, orientación sexual, identidad de género y esquemas de género del autoconcepto en bailarines: una revisión sistemática

\title{
RESUMEN
}

El concepto de imagen corporal está basado en un constructo multidimensional, formado de las representaciones sobre el tamaño y apariencia del cuerpo. Se objetivó verificar de forma sistematizada la literatura estudios que presentaran la relación entre satisfacción de la imagen corporal y variables sexuales. Los resultados demostraron que el área que estudia la percepción de la imagen corporal en bailarines aún no percibió la importancia de otras dimensiones como identidad de género, orientación sexual y los esquemas de género del autoconcepto, que pueden tener mayor impacto que sólo el criterio sexual biológico. El sexo biológico sigue siendo la principal variable de control en la comparación en los estudios entre los bailarines, siendo urgente la necesidad de otras dimensiones de la identidad a ser incorporadas en los próximos estudios, como las citadas arriba, para ampliar la capacidad de explicar la satisfacción corporal humana.

PALABRAS-CLAVE: Imagen corporal; El sexo; Orientación sexual; Identidad de género 


\section{INTRODUÇÃO}

A imagem corporal é um construto multidimensional, definido como o conceito de si próprio em relação ao outro (ADAMI et al., 2005). Este fenômeno também pode ser definido como processos cognitivos, emocionais, fisiológicos e sociais, que estão em constantes alterações (SIMAS et al., 2014). Essas mudanças são influenciadas a partir de variáveis como o sexo biológico, idade, crenças, valores e comportamentos referentes à cultura no qual o indivíduo esteja inserido (ALMEIDA et al., 2005; FERREIRA; LEITE, 2002).

Nesse sentido, é possível perceber a imagem corporal como um fator influenciador das atividades da vida humana, intervindo nos processos de escolha, partindo desde as roupas, vestimentas ou adornos, bem como os interesses estéticos e as habilidades de empatizar as emoções do outro (RIBEIRO; OLIVEIRA, 2011). Sendo assim, a partir dessas escolhas o indivíduo passa a moldar a sua percepção sobre a sua autoimagem, podendo estar satisfeito ou não com a própria imagem corporal.

Deste modo, a satisfação com a imagem corporal pode ser percebida de formas diferentes, quando comparada entre o sexo biológico, os homens/machos almejam um corpo musculoso, ou seja, com grande volume muscular, e em sua maioria apresentam insatisfação pela falta de massa corporal (MANTOVANI et al., 2009), enquanto as mulheres/fêmeas em sua maioria, buscam um corpo magro, priorizando uma cintura mais fina, quadris largos e seios avantajados, e demonstram insatisfação pelo excesso de massa corporal (MATSUDO et al., 2009; REBOLHO et al., 2014; DE AZEVENDO et al., 2017)

Essas diferenças também têm sido evidenciadas em relação à orientação sexual, e identidade de gênero, em que os homens homossexuais apontam insatisfação com a sua autoimagem pela falta de massa corporal (TEIXEIRA et al., 2013), tendo em vista a figura do homem/macho como uma identidade de gênero tipicamente masculina tem maior valor nos ambientes homossexuais.

Nesse sentido, diversos estudos estão sendo realizados com objetivo de verificar essas diferenças em grupos e contextos específicos, como atletas (FORTES et al., 2013), crianças e adolescentes (MOCKDECE et al., 2017). No contexto da dança a preocupação com a imagem corporal é evidenciada tanto em homens quanto em mulheres, que vivem em constante busca por uma imagem corporal perfeita e ideal (HAAS, et al., 2010; RIBEIRO; DA VIEGA, 2010; REIS et al., 2014). No entanto, essas comparações em bailarinos têm sido realizadas somente em relação ao sexo biológico.

Nessa perspectiva, a dança pode ser observada como uma prática socialmente instruída, na qual o corpo do indivíduo/bailarino está associado à sua identidade de gênero, ou seja, o corpo é 
observado dentro das várias modalidades de como um parâmetro de normatização e aplicação das normas de gênero, estimulando diversos tipos de corpos, sejam eles masculinos ou femininos e andróginos (ANDREOLI, 2010).

Assim os indivíduos que praticam determinada modalidade, são estereotipados por aquelas características, fortemente relacionadas aos sexos, sendo muitas vezes classificados com uma orientação sexual divergente da sua, por estar em uma modalidade que rompe com as barreiras de gênero entre homens e mulheres (MELO et al., 2015).

Nesse sentido, o objetivo desta pesquisa é verificar de forma sistematizada a literatura buscando estudos que apresentem comparações da satisfação da imagem corporal entre as variáveis: sexo biológico, identidade de gênero, orientação sexual e esquemas de gênero do autoconceito em bailarinos.

\section{MÉTODO}

O presente estudo apresenta uma revisão sistemática de literatura, com o intuito de identificar, selecionar, extrair dados, analisar e avaliar criteriosamente todos os estudos que abordem a satisfação da imagem corporal em relação ao sexo biológico, orientação sexual, identidade de gênero e esquema de gênero do autoconceito em bailarinos, seguindo rigorosamente métodos sistemáticos claros em cada etapa realizada. A revisão foi realizada no ano de 2018 , em sete conceituadas bases de dados eletrônicas, PubMed, Scopus, PsycINFO, Scielo, Web of Science e Lilacs. Não foi definido período para publicações, a fim de buscar o máximo de estudos dentro da literatura, porém estabeleceu-se o limite dos estudos publicados até dezembro de 2018, nas línguas Portuguesa, Inglesa e Espanhola.

Foram incluídos estudos que apresentaram textos completos com amostra de bailarinos. Excluiu-se da pesquisa os estudos caracterizados metodologicamente como revisões sistemáticas ou de literatura, estudos-piloto, estudo de caso, estudos de protocolos, dissertações, teses, capítulos de livros, artigos repetidos, artigos sem pertinência (que fogem do objetivo principal proposto no estudo, e que não descreveram com clareza o método utilizado no estudo).

Os descritores utilizados foram pré-selecionados nos Descritores em Ciências da Saúde (DeCS) da BVS. Sendo aplicados os seguintes blocos de pesquisa: Body Image/ Satisfaction body/, dancers/ gender identity/ role of Gender/ Gender Psychological Profile, seguindo as seguintes estratégias de busca: (“body image”) OR ("satisfaction image”) OR (“dancers") OR ("Gender Identity") OR ("Role of Gender") and (“Gender Psychological Profile”). 
O processo de busca foi realizado separadamente, por dois pesquisadores, que ao fazer as buscas nas bases de dados averiguavam o número exato de estudos encontrados e os compararam, a fim de obter os mesmos resultados encontrados. Com os estudos já selecionados deu-se início a leitura dos títulos, elencando os artigos que seguiriam no processo de seleção. Em casos de divergência nos títulos selecionados entre pesquisadores, um terceiro pesquisador realizou a leitura dos títulos e tomou a decisão final.

Posteriormente, os estudos foram submetidos a análises qualitativas e quantitativas, utilizando o instrumento adaptado de Ursi (2005), que mostra de fato os dados a serem extraídos e analisados, buscando organizar e categorizar as informações de forma mais precisa, incluído principalmente título do estudo, ano, principais resultados e conclusões. Bem como o Strengthening the Reporting of Observational Studies in Epidemiology (STROBE), o qual é constituído por 22 itens com pontuação de (zero) a 1, que analisa a qualidade metodológica dos estudos selecionados. Os itens que constituem o STROBE são relacionados às informações que devem estar presentes na elaboração do estudo, tais como o título, resumo, introdução, metodologia, resultados e discussão (VON ELM et al., 2008).

Para melhor avaliar a qualidade dos artigos a pontuação do instrumento foi transformada em percentual. Estabelecendo-se três categorias para avaliação da qualidade: A - quando o estudo atende mais de $80 \%$ dos critérios estabelecidos; B - quando 50-79\% dos critérios foram preenchidos, e $\mathrm{C}$ - quando menos de $49 \%$ dos critérios foram preenchidos.

\section{RESULTADOS}

As buscas resultaram em um total de 237 artigos, sendo incluídos ao final da revisão 4 estudos, o processo de revisão com a exclusão dos artigos pelo título, resumo e palavras chaves, como mostra a Figura 1. 
Figura 1 - Fluxograma de identificação e seleção dos artigos para revisão sistemática

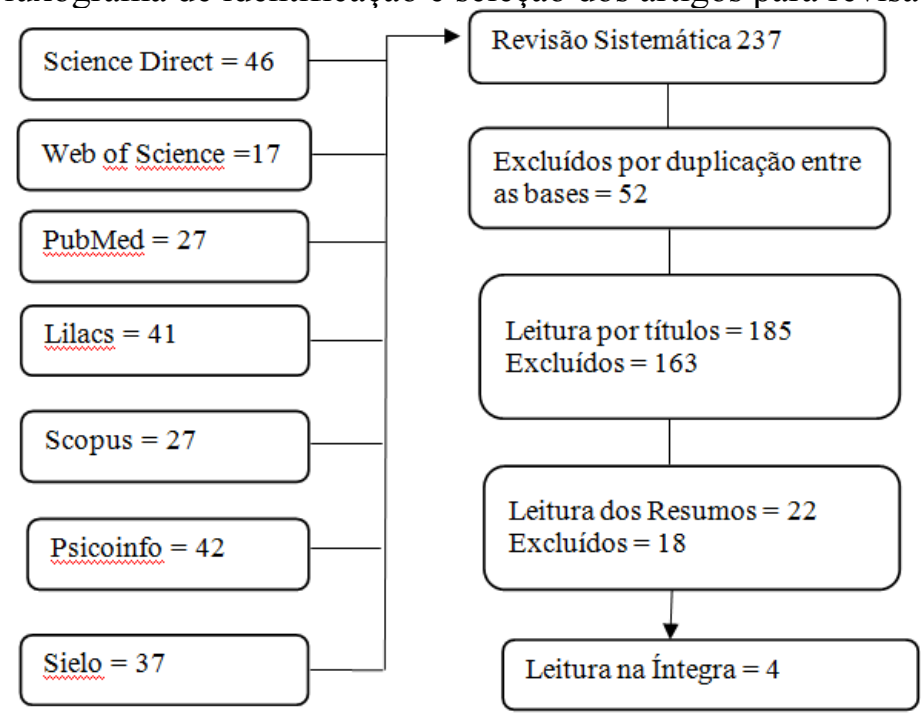

Fonte: Elaborado pelo autor (2018)

$\mathrm{Na}$ tabela 1 são apresentadas as principais características metodológicas dos 4 estudos finais que fazem parte dessa revisão, contendo o autor, ano, título, local do estudo, população, sexo, média de idade, fator de impacto e o periódico onde foi publicado.

Tabela 1. Principais características dos estudos

\begin{tabular}{|c|c|c|c|c|c|}
\hline $\begin{array}{c}\text { Autor / } \\
\text { Ano }\end{array}$ & Título & $\begin{array}{l}\text { Local } \\
\text { de } \\
\text { Estudo }\end{array}$ & $\begin{array}{l}\text { População } \\
\text { (n, e Média } \\
\text { de Idade) }\end{array}$ & $\begin{array}{c}\text { Fator } \\
\text { de } \\
\text { Impact } \\
\text { o }\end{array}$ & Periódico \\
\hline $\begin{array}{l}\text { Ravaldi et } \\
\text { al. } 2003\end{array}$ & $\begin{array}{l}\text { Eating Disorders and Body } \\
\text { Image Disturbances among } \\
\text { Ballet Dancers, Gymnasium } \\
\text { Users and Body Builders }\end{array}$ & Itália & $\begin{array}{c}113 \mathrm{BN} \\
16.2 \pm 4.1\end{array}$ & NI & Psychopathology \\
\hline $\begin{array}{l}\text { Hartmann } \\
\text { et al. } 2017\end{array}$ & $\begin{array}{lcr}\begin{array}{l}\text { Sintomas } \\
\text { alimentares }\end{array} & \text { de } & \text { transtornos } \\
\text { profissionais } & & \text { bailarinos } \\
& & \end{array}$ & Brasil & $\begin{array}{c}35 \mathrm{BP}, 22,2 \\
\quad \pm 6,8 .\end{array}$ & $\begin{array}{l}\text { SJR } \\
0.22\end{array}$ & $\begin{array}{l}\text { Nutrición Clínica } \\
\text { Dietética } \\
\text { Hospitalaria }\end{array}$ \\
\hline $\begin{array}{c}\text { Reis et al. } \\
2013\end{array}$ & $\begin{array}{lcr}\text { Imagem } & \text { corporal, estado } \\
\text { nutricional } & \text { e sintomas } & \text { de } \\
\text { transtornos } & \text { alimentares } & \text { em } \\
\text { bailarinos } & & \end{array}$ & Brasil & $\begin{array}{l}156 \mathrm{BP}, \mathrm{NI} \\
21,7 \pm 20,3\end{array}$ & NI & $\begin{array}{l}\text { Revista Brasileira } \\
\text { de Atividade } \\
\text { Física \& Saúde }\end{array}$ \\
\hline $\begin{array}{l}\text { Ravaldi et } \\
\text { al. } 2006\end{array}$ & $\begin{array}{l}\text { Gender role, eating disorder } \\
\text { symptomos, and body image } \\
\text { concern in ballet dancers. }\end{array}$ & Itália & $\begin{array}{c}110 \mathrm{BP}, 59 \\
\mathrm{GC} \\
16,5 \pm 17,4\end{array}$ & $\begin{array}{c}\text { JCR } \\
1.954\end{array}$ & $\begin{array}{l}\text { Journal of } \\
\text { Psychosomatic } \\
\text { Research }\end{array}$ \\
\hline
\end{tabular}

$\mathrm{BN}=$ Bailarinos não profissionais; $\mathrm{BP}=$ Bailarinos profissionais; $\mathrm{GC}=$ Grupo Controle; $\mathrm{PD}=\mathrm{Pole}$ Dance; $\mathrm{U}=$ Universitárias.

Os resultados apontam que as publicações foram realizadas de 2003 a 2017. O estudo com maior número de participantes foi o conduzido por Reis et al. (2013) com 156 participantes, enquanto com menor número foi no estudo realizado por Hartmann et al. (2017), contando apenas 
com 35 participantes. Entre os bailarinos a idade mínima encontrada foi de 16 anos, enquanto que a maior foi 22 anos. Os estudos foram realizados em dois países: Brasil e Itália.

Na tabela 2 apresenta-se os objetivos, instrumentos e principais resultados encontrados nos estudos incluídos na revisão, além disso é apresentada a classificação quanto a qualidade de cada estudo por meio do STROBE.

Tabela 2. Objetivos, instrumentos, principais resultados e avaliação da qualidade metodológica dos estudos incluídos na revisão.

\begin{tabular}{|c|c|}
\hline Objetivos & $\begin{array}{l}\text { Instrumentos } \\
\text { utilizados }\end{array}$ \\
\hline $\begin{array}{l}\text { Avaliar o grau de } \\
\text { insatisfação da imagem } \\
\text { corporal e distúrbios } \\
\text { alimentares. }\end{array}$ & $\begin{array}{l}\text { Beck Depression } \\
\text { Inventory (BDI), State- } \\
\text { Trait Anxiety Inventory } \\
\text { (IDATE) e Corpo } \\
\text { Uneasiness Test }(M A S)\end{array}$ \\
\hline $\begin{array}{l}\text { Conhecer a frequência } \\
\text { do comportamento } \\
\text { alimentar anormal e } \\
\text { insatisfação da imagem } \\
\text { corporal de bailarinos } \\
\text { profissionais de uma } \\
\text { companhia de ballet. }\end{array}$ & $\begin{array}{l}\text { Eating Attitudes Test } \\
\text { (EAT-26), Bulimic } \\
\text { Investigatory } \quad \text { Test } \\
\text { Edinburgh (BITE), Body } \\
\text { Shape Questionnarie } \\
\text { (BSQ) }\end{array}$ \\
\hline $\begin{array}{lr}\text { Analisar a percepção de } \\
\text { imagem corporal, estado } \\
\text { nutricional e sintomas } \\
\text { de } & \text { transtornos } \\
\text { alimentares } & \text { de } \\
\text { bailarinos. } & \end{array}$ & $\begin{array}{l}\text { Escala de silhuetas } \\
\text { proposta por Stunkard, } \\
\text { Sorenson e Schlusinger; } \\
\text { O Teste de Atitudes } \\
\text { Alimentares (EAT-26); } \\
\text { Bulimic Inventory Test } \\
\text { Edinburgh (BITE); }\end{array}$ \\
\hline
\end{tabular}

Avaliar a relação entre o papel de gênero, comportamento alimentar e imagem corporal em bailarinas não profissionais.

Bem Sex Role Inventory (BSRI); Eating Disorder Examination (EDE); Body Uneasiniss Test (BUT); Beck Depression Inventory (BDI).
Principais resultados

Bailarinas não profissionais apresentaram maior prevalência de distúrbios alimentares (anorexia nervosa 1,8\%; bulimia nervosa $2,7 \%$; desordens sem outra especificação de $22,1 \%$ de comer)

Risco de desenvolvimento de anorexia no sexo feminino, $75 \%$ do sexo feminino apresentou comportamentos bulímico e $14 \%$ no sexo masculino. A distorção da imagem corporal foi encontrada apenas no sexo feminino, em $75 \%$ das bailarinas.

A prevalência de insatisfação da imagem corporal foi de $72 \%$ em que homens encontram-se insatisfeitos pela magreza e as mulheres insatisfeitas pelo excesso de peso. Os bailarinos ainda presentaram sintomas de anorexia e bulimia nervosa, a associação negativa foi observada pela magreza, sexo e sintomas de bulimia nervosa.

Os bailarinos apontam ter uma maior distorção da imagem corporal e possíveis distúrbios alimentares em relação ao grupo controle. Ainda nesse estudo os bailarinos em sua maioria apresentaram um papel indiferenciado de gênero.
Strob

$\mathbf{e}$

B

B

B

B

De modo geral todos os estudos buscaram avaliar a imagem corporal, bem como os distúrbios e transtornos alimentares nos bailarinos. Os artigos 1, 2 e 3, compararam a satisfação com a imagem corporal somente entre os sexos. Por outro lado, o artigo 3, realizado por Ravaldi et al., (2006), apresentou seus resultados comparando a satisfação com a imagem corporal entre identidade de gênero, classificando as bailarinas em masculinas, femininas, andróginas e indiferenciadas. Os artigos 2 e 3 utilizaram do mesmo instrumento Bulimic Inventory Test Edinburgh (BITE) para avaliar os transtornos alimentares em suas pesquisas. Ao observar a 
qualidade metodológica dos estudos, verificou-se que todos as pesquisas que constituem esta revisão foram classificadas com a categoria $\mathrm{B}$, em que $50-79 \%$ dos critérios foram preenchidos.

\section{DISCUSSÃO}

O objetivo do presente estudo foi verificar de forma sistematizada a literatura buscando estudos que realizaram comparações da satisfação da imagem corporal entre as variáveis como sexo biológico, identidade de gênero, orientação sexual e esquemas de gênero do autoconceito em bailarinos. Em vista disso, percebe-se que profissionalmente no mundo da dança, o bailarino passa por um processo de formação desde o início de sua carreira, a disciplina, dedicação e perfeccionismo passam a ser presente em todos os sentidos, principalmente quando se trata da forma física, uma vez que a busca pelo corpo perfeito é uma busca diária (BOLLING; PINHEIRO, 2010). Assim, avaliando os resultados apontados pelos estudos incluídos nesta revisão é constatado que bailarinos profissionais, independentemente da modalidade, apresentam insatisfação com a imagem corporal, sendo que a maior insatisfação está presente nas mulheres/ fêmeas.

Neste sentido, o estudo realizado por Ravaldi et al., (2003), ao avaliarem os transtornos alimentares e distúrbios da imagem corporal entre bailarinos, usuários de ginásio e praticantes de fisiculturismo, revelou que o grupo de bailarinas apontou maior insatisfação com sua própria imagem corporal, em relação aos grupos presentes no estudo. Os autores ainda apontam que a insatisfação com a imagem corporal está presente em todos os grupos pesquisados, porém com diferentes níveis e graus de insatisfação, o grupo controle, constituído por não praticantes de nenhum tipo de atividade física, demonstrou que o mal-estar com o próprio corpo está diretamente relacionado com o peso corporal. Já nos fisiculturistas a insatisfação está relacionada com falta de massa corporal.

Em relação aos bailarinos a insatisfação com a imagem corporal está diretamente relacionada com o excesso de massa corporal, já que eles sofrem pressão por parte das escolas de ballet, com o intuito de propor um padrão de corpo perfeito e ideal como sinônimo de sucesso, bem como a influência dos pais, para que seus filhos busquem o sucesso e um bom desempenho no meio artístico.

Com relação à comparação da percepção da imagem corporal de bailarinos entre o sexo biológico Hartmann et al., (2017), apontam que as bailarinas apresentam maior distorção da imagem corporal, risco de desenvolvimento de anorexia e comportamento bulímico quando comparadas aos homens. Ao encontro desse achado, Reis et al., (2013), ao investigarem 156 bailarinos, verificaram que $72 \%$ apresentaram insatisfação com a imagem corporal, sendo que os 
homens/machos possuíam insatisfação pela falta de massa corporal e as mulheres/fêmeas pelo excesso de massa corporal.

De forma mais específica, o estudo conduzido por Ravaldi et al., (2006) investigou as relações entre o papel de gênero, comportamento alimentar e distúrbios da imagem corporal em 110 bailarinas do sexo feminino, e 59 estudantes. De modo geral, as bailarinas pontuaram mais do que as estudantes nos itens que avaliavam a percepção da imagem corporal e o comportamento alimentar. Por outro lado, observou-se que em suma as bailarinas se classificaram com papel de gênero indiferenciado, porém as bailarinas tipificadas como masculinas apresentaram maiores níveis de sintomas de desordem alimentar e insatisfação corporal.

A partir dos estudos incluídos na presente revisão, pode-se perceber que o enfoque das pesquisas na área da imagem corporal no contexto das danças, está nas associações deste construto com distúrbios e transtornos alimentares. Observa-se também, que essas associações se dão somente em função da variável sexo biológico, o que explica uma pequena parte desse construto, tendo em vista que, outros fatores podem ser determinantes para a percepção da imagem corporal. Morrinson et al., (2004) por exemplo realizaram uma meta-análise de 27 estudos que investigavam a orientação sexual e um aspecto específico da imagem corporal, a satisfação corporal, isto é, quanto gays e lésbicas diferiam de heterossexuais masculinos e femininos em termos de atitude e comportamento corporal.

Seus resultados indicam que gays aparentam ser mais propensos a ter insatisfação com a sua imagem corporal que heterossexuais masculinos, e um número menor de estudos apontou que lésbicas parecem ser ligeiramente mais satisfeitas com seus corpos do que heterossexuais femininas. Afirma ainda que, no universo da satisfação corporal, a orientação sexual parece influenciar mais os homens do que as mulheres. Deste modo, nota-se que a orientação sexual, bem como outras variáveis relacionadas à identidade sexual pode ajudar em uma melhor compreensão do fenômeno imagem corporal.

\section{CONSIDERAÇÕES FINAIS}

Compreender o universo dos bailarinos é compreender as inquietações presentes no seu dia-a-dia. A imagem corporal é um fator que requer atenção por parte dos bailarinos, das escolas de dança e até mesmo da família. Nesse sentido, buscou-se assimilar as relações entre percepção da imagem corporal com o sexo biológico, orientação sexual, identidade de gênero e os esquemas de gênero do autoconceito em bailarinos especificamente e muito pouco se encontrou. 
Os resultados dessa revisão demonstram que área que estuda a percepção da imagem corporal em bailarinos ainda não percebeu a importância de outras dimensões da Identidade (identidade de gênero, orientação sexual e os esquemas de gênero do autoconceito), que podem ter maior impacto do que apenas o critério sexo biológico. O sexo biológico ainda é a principal variável de controle na comparação nos estudos entre os bailarinos, sendo urgente a necessidade de outras dimensões da identidade incorporadas nos próximos estudos, como as citadas acima, para ampliar a capacidade de explicarmos a satisfação corporal humana.

Quanto à qualidade metodológica dos estudos avaliados pela escala STROBE, nota-se que a maioria foram classificados como bons, apesar de terem sidos realizados com um número relevantes de bailarinos participantes. Os estudos não obtiveram classificação máxima porque não apresentaram informações como: não explicou o método de seleção dos participantes e nem definiu os critérios de tamanho amostral, não explicaram como foram tratadas as variáveis quantitativas na análise dos estudos, não descreveram as categorias de análises que foram adotadas, não apresentaram estimativas estatísticas não ajustadas e não expuseram as estimativas ajustadas por variáveis confundidoras e suas respectivas precisões, não deixaram claro quais foram as variáveis confundidoras utilizadas na análise ajustada e o motivo por terem sido incluídas e, por último não especificaram a fonte de financiamento dos estudos e o respectivo papel dos financiadores. Também não informaram o ponto de corte utilizado para categorizar variáveis contínuas e não transformaram as estimativas de risco relativo em termos de risco absoluto, para um período de tempo relevante. Desse modo, nem um dos estudos explicou a percepção da imagem corporal em sua totalidade, limitando-se a explicá-la apenas pelo sexo biológico, mas por outras variáveis.

Dessa forma, sugere-se que novos estudos sejam realizados com essa população, tendo em vista, os dados alarmantes de insatisfação corporal atrelado aos transtornos alimentares, dando ênfase em outras variáveis a serem controladas, como a orientação sexual, as identidades de gênero, tais quais os esquemas de gênero do autoconceito que poderão estruturar novas hipóteses.

\section{REFERÊNCIAS}

ADAMI, Fernando et al. Aspectos da construção e desenvolvimento da imagem corporal e implicações na educação física. Revista Digital de Buenos Aires, v. 83, 2005. Disponível em: https://www.efdeportes.com/efd83/imagem.htm. Acesso em: $12 \mathrm{dez} 2018$.

DE ALMEIDA, Graziela Aparecida Nogueira et al. Percepção de tamanho e forma corporal de mulheres: Estudo exploratório. Psicologia em estudo, v. 10, n. 1, p. 27-35, 2005. Disponível em: http://www.scielo.br/pdf/\%0D/pe/v10n1/v10n1a04.pdf. Acesso em 20 de dez 2018. 
ANDREOLI, Giuliano Souza. Dança, gênero e sensualidade: um olhar cultural.

CONJECTURA: filosofia e educação, v. 15, n. 1, 2010. Disponível em:

http://www.ucs.com.br/etc/revistas/index.php/conjectura/article/view/186. Acesso em: 11 dez 2018.

BOLLING, Caroline Silveira; PINHEIRO, Tarcisio Marcio Magalhães. Bailarinos profissionais e saúde: uma revisão da literatura. 2010. Disponível em:

https://www.researchgate.net/profile/Caroline_Bolling/publication/320760871_Professional_dancer s_and_health_a_literature_review/links/59f9cdfbaca27221807e8f5c/Professional-dancers-andhealth-a-literature-review.pdf. Acesso em: 01 dez. 2018

DE AZEVEDO PAIVA, Adriana et al. Percepção da imagem corporal e estado nutricional em acadêmicas de nutrição de uma universidade pública. DEMETRA: Alimentação, Nutrição \& Saúde, v. 12, n. 1, p. 193-206, 2017. Disponível em: https://www.epublicacoes.uerj.br/index.php/demetra/article/view/22483. Acesso em 10 dez 2018.

FERREIRA, Maria Cristina; LEITE, Neíse Gonçalves de Magalhães. Adaptação e validação de um instrumento de avaliação da satisfação com a imagem corporal. Avaliação Psicológica: Interamerican Journal of Psychological Assessment, v. 1, n. 2, p. 141-149, 2002. Disponível em: https://dialnet.unirioja.es/servlet/articulo?codigo=5262560. Acesso em $12 \mathrm{dez} 2019$.

FORTES, Leonardo de Sousa; ALMEIDA, Sebastião de Sousa; FERREIRA, Maria Elisa Caputo. Body image dissatisfaction in sports of masculine gender. Jornal Brasileiro de Psiquiatria, v. 62, n. 2, p. 101-107, 2013. Disponível em: http://www.scielo.br/scielo.php?pid=S0047$20852013000200002 \&$ script=sci_arttext\&tlng=pt. Acesso em $10 \mathrm{dez} 2018$.

HAAS, Aline Nogueira; GARCIA, Anelise Cristina Dias; BERTOLETTI, Juliana. Imagem corporal e bailarinas profissionais. Revista Brasileira de Medicina do Esporte. vol. 16, n. 3 (maio/jun. 2010), p. 182-185, 2010. Disponível em:

https://www.lume.ufrgs.br/handle/10183/72043. Acesso em: 02 dez 2018

HARTMANN, Lívia Maciel et al. Sintomas de transtornos alimentares em bailarinos profissionais. Nutrición clínica y dietética hospitalaria, v. 37, n. 3, p. 151-157, 2017. Disponível em: https://dialnet.unirioja.es/servlet/articulo?codigo=618925. Acesso em $11 \mathrm{dez} 2018$.

MANTOVANI, B. et al. Nível de satisfação com a imagem corporal de universitários [Internet]. Buenos Aires 2009. Disponível em: http://www.efdeportes.com. Acesso em: 13 dez 2018.

MATSUO, Renata Frazão et al. Imagem corporal de idosas e atividade física. Revista Mackenzie de Educação Física e Esporte, v. 6, n. 1, 2009. Disponível em:

http://editorarevistas.mackenzie.br/index.php/remef/article/view/1281. Acesso em 03 de dez 2018.

MELO, Gislane Ferreira et al. Estereótipos de gênero aplicados a homens atletas praticantes de esportes culturalmente femininos: a percepção de leigos, profissionais da Educação Física e atletas profissionais. Revista Brasileira de Ciência e Movimento, v. 23, n. 3, p. 30-37, 2015. Disponível em: https://portalrevistas.ucb.br/index.php/RBCM/article/view/5408. Acesso em 05 dez 2018.

MENDES, Karina Dal Sasso; SILVEIRA, Renata Cristina de Campos Pereira; GALVÃO, Cristina Maria. Revisão integrativa: método de pesquisa para a incorporação de evidências na saúde e 
na enfermagem. Texto \& contexto enfermagem, v. 17, n. 4, p. 758-764, 2008. Disponível em: https://www.redalyc.org/pdf/714/71411240017.pdf. Acesso em 10 dez 2018.

MORRISON, Melanie A.; MORRISON, Todd G.; SAGER, Cheryl-Lee. A satisfação corporal é diferente entre homens gays e mulheres lésbicas e homens e mulheres heterossexuais? : Uma revisão meta-analítica. Imagem corporal, v. 1, n. 2, p. 127-138, 2004. Disponível em: https://www.sciencedirect.com/science/article/abs/pii/S1740144504000282. Acesso em $10 \mathrm{dez}$ 2018.

NEVES, Clara Mockdece et al. Imagem corporal na infância: uma revisão integrativa da literatura. Revista Paulista de Pediatria: Órgão Oficial da Sociedade de Pediatria de São Paulo, v. 35, n. 3, p. 331-339, 2017. Disponível em: http://www.scielo.br/pdf/rpp/2017nahead/0103-0582rpp-2017-35-3-00002.pdf. Acesso em: 07 jan 2018.

RAVALDI, Claudia et al. Gender role, eating disorder symptoms, and body image concern in ballet dancers. Journal of psychosomatic research, v. 61, n. 4, p. 529-535, 2006. Disponível em: https://www.sciencedirect.com/science/article/pii/S0022399906002212 Acesso em 02 dez 2018.

RAVALDI, Claudia et al. Distúrbios alimentares e distúrbios da imagem corporal em bailarinas, usuários de academias e fisiculturistas. Psicopatologia, v. 36, n. 5, p. 247-254, 2003. Disponível em: https://www.karger.com/Article/Abstract/73450. Acesso em 10 dez 2018.

REBOLHO MARTINS, Cilene et al. Insatisfação com a imagem corporal e fatores associados em universitários. Estudos de psicologia, v. 17, n. 2, 2012. Disponível em: https://www.redalyc.org/html/261/26124614007/. Acesso em 05 dez 2018.

REIS, Nycolle et al. Imagem corporal, estado nutricional e sintomas de transtornos alimentares em bailarinos. Revista Brasileira de Atividade Física \& Saúde, v. 18, n. 6, p. 771-771, 2013. Disponível em: http://rbafs.org.br/RBAFS/article/view/3043. Acesso em 04 jan 2018.

RIBEIRO, Lena Guimarães; VEIGA, Gloria Valeria da. Imagem corporal e comportamentos de risco para transtornos alimentares em bailarinos profissionais. Rev Bras Med Esporte, v. 16, $\mathrm{n}$. 2, p. 99-102, 2010. Disponível em: https://submission3.scielo.br/index.php/rbme/article/view/14198 Acesso em: 19 dez 2018.

SIMAS, Joseani Paulini Neves; MACARA, Ana; MELO, Sebastião Iberes Lopes. Imagem corporal e sua relação com peso e índice de massa corporal em bailarinos profissionais. Rev. bras. med. esporte, v. 20, n. 6, p. 433-437, 2014. Disponível em:

http://www.scielo.br/scielo.php?pid=S1517-6922014000600433\&script=sci_abstract\&tlng=pt. Acesso em: 08 jan 2018.

TEIXEIRA, Fabiano Augusto et al. Insatisfação com a imagem corporal e fatores associados em homens homossexuais. Revista Brasileira de Ciência e Movimento, v. 23, n. 4, p. 46-56, 2015. Disponível em: https://portalrevistas.ucb.br/index.php/RBCM/article/view/5596. Acesso em:20 jan 2018.

URSI, Elizabeth Silva; GAVÃO, Cristina Maria. Prevenção de lesões de pele no perioperatório: revisão integrativa da literatura. Revista Latino-Americana de Enfermagem, v. 14, n. 1, p. 124131, 2006. Disponível em: https://www.redalyc.org/pdf/2814/281421858017.pdf. Acesso em: 22 de jan 2018. 
VANDENBROUCKE, Jan P. et al. Fortalecimento do Relatório de Estudos Observacionais em Epidemiologia (STROBE): explicação e elaboração. PLoS medicine, v. 4, n. 10, p. e297, 2007. Disponível em: https://journals.plos.org/plosmedicine/article?id=10.1371/journal.pmed.0040297 Acesso em: 11 de jan 2018.

VON ELM, Erik et al. Declaración de la Iniciativa STROBE (Strengthening the Reporting of Observational studies in Epidemiology): directrices para la comunicación de estudios observacionales. Gaceta Sanitaria, v. 22, n. 2, p. 144-150, 2008. Disponível em:

https://www.sciencedirect.com/science/article/pii/S0213911108712238. Acesso em: 10 jan 2018.

\section{NOTAS DE AUTOR}

\section{AGRADECIMENTOS}

Agradecimento ao Instituto Werner/IPVESC e a Coordenação de Aperfeiçoamento de Pessoal de Nível Superior.

\section{CONTRIBUIÇÃO DE AUTORIA}

Concepção do manuscrito: A. C. Brandão, K. T. D. Freitas, H. P. De Carvalho

Coleta de dados: A. C. Brandão, K. T. D. Freitas, H. P. De Carvalho

Análise de dados: A. C. Brandão, K. T. D. Freitas, W.R. Da Silva

Discussão dos resultados: A. C. Brandão, K. T. D. Freitas, H. P. De Carvalho, F.L. Cardoso

Produção do texto: A. C. Brandão, F.L. Cardoso

Revisão e aprovação: A. C. Brandão, F.L. Cardoso

FINANCIAMENTO

Não se aplica.

\section{CONSENTIMENTO DE USO DE IMAGEM}

Não se aplica.

\section{APROVAÇÃO DE COMITÊ DE ÉTICA EM PESQUISA}

Não se aplica.

\section{CONFLITO DE INTERESSES}

Não há conflitos de interesses.

\section{LICENÇA DE USO}

Os autores cedem à Motrivivência - ISSN 2175-8042 os direitos exclusivos de primeira publicação, com o trabalho simultaneamente licenciado sob a Licença Creative Commons Attribution Non-Comercial ShareAlike (CC BY-NC SA) 4.0 International. Esta licença permite que terceiros remixem, adaptem e criem a partir do trabalho publicado, desde que para fins não comerciais, atribuindo o devido crédito de autoria e publicação inicial neste periódico desde que adotem a mesma licença, compartilhar igual. Os autores têm autorização para assumir contratos adicionais separadamente, para distribuição não exclusiva da versão do trabalho publicada neste periódico (ex.: publicar em repositório institucional, em site pessoal, publicar uma tradução, ou 
como capítulo de livro), com reconhecimento de autoria e publicação inicial neste periódico, desde que para fins não comerciais e compartilhar com a mesma licença.

\section{PUBLISHER}

Universidade Federal de Santa Catarina. Programa de Pós-Graduação em Educação Física. LaboMídia - Laboratório e Observatório da Mídia Esportiva. Publicado no Portal de Periódicos UFSC. As ideias expressadas neste artigo são de responsabilidade de seus autores, não representando, necessariamente, a opinião dos editores ou da universidade.

\section{EDITORES}

Mauricio Roberto da Silva, Giovani De Lorenzi Pires, Rogério Santos Pereira

\section{HISTÓRICO}

Recebido em: 06 de abril de 2019.

Aprovado em: 06 de junho de 2019. 